\title{
Sistema de excitación por pulsos para la caracterización de resonadores para atomización
}

\author{
X. JORDÀ', M. LOZANO ${ }^{1}$, E. CHICO ${ }^{1}$, A. LOZANO ${ }^{2}$, H. AMAVEDA ${ }^{3}$, F. BARRERA ${ }^{3}$ \\ 1 CNM-IMB (CSIC). Campus UAB. 08193 Bellaterra, Barcelona \\ ${ }^{2}$ LITEC (CSIC), 50015 Zaragoza \\ ${ }^{3}$ CPS, Universidad de Zaragoza, 50015 Zaragoza
}

\begin{abstract}
El desarrollo de materiales piezocerámicos ha despertado un renovado interés en el campo de la atomización para generar gotas de tamaño microscópico, difíciles de conseguir por los medios mecánicos habituales. En este trabajo se presenta un sistema de excitación de cerámicas piezoeléctricas encaminado a su caracterización, basado en un convertidor DC/AC resonante con topología de puente completo que permite trabajar hasta $2 \mathrm{MHz}$. La salida del convertidor, aplica una onda de tensión cuadrada bajo la forma de ráfagas de impulsos, limitando el autocalentamiento de las cerámicas testeadas. Las señales de control permiten fijar todos los parámetros de test: frecuencia de resonancia, número de impulsos de salida así como frecuencia de repetición, ciclo de trabajo y tensión pico a pico de los mismos. Los primeros ensayos realizados sobre una cerámica de 1,668 MHz han permitido observar la lógica dependencia de la eficacia de atomización con la frecuencia de excitación y han puesto de manifiesto la importancia del ciclo de trabajo, del número mínimo de impulsos para iniciar la atomización y de su valor pico a pico de tensión que genera distintas distribuciones en diámetro de gota, aunque siempre con un máximo situado alrededor de $9 \mu \mathrm{m}$.
\end{abstract}

Palabras clave: Atomización, cerámica piezoeléctrica, resonador piezoeléctrico, convertidor resonante.

\section{Impulsional excitation system for resonator characterisation in atomization applications}

Piezoelectric ceramic materials are of main interest in the field of spray-dryng applications, in order to generate microscopic droplets which are difficult to obtain by mechanical systems. In this work an excitation electronic system for piezoelectric transducers is presented. The system is oriented to device characterization with resonance frequencies up to $2 \mathrm{MHz}$ and is based on a full bridge DC - AC resonant voltage converter. The converter applies a square voltage waveform burst, limiting the device under test self-heating. The following test parameters can be adjusted: frequency, duty cycle and peak-to-peak voltage of the output waveform, number of output pulses constituting the burst and its repetition rate. First results with a $1.668 \mathrm{MHz}$ ceramic disc show the spray-dryng efficiency dependence on the excitation frequency, but also on the duty cycle, minimum number of pulses to start the spray-dryng process and peak-to-peak voltage. This last variable modifies the droplet diameter distribution but not the peak value, centered at $9 \mu \mathrm{m}$.

Keywords: Spray-drying, piezoelectric ceramics, piezoelectric resonator, resonant converter.

\section{INTRODUCCIÓN}

La atomización ultrasónica es un método eficiente para generar aerosoles de tamaños de gota microscópicos, difíciles de conseguir mediante los medios mecánicos habituales (atomizadores de presión o asistidos por flujos gaseosos). Para ello se utilizan alternativamente dos métodos: hacer circular el flujo a través de una onda ultrasónica estacionaria [1], o depositar el volumen del fluido sobre la superficie de un transductor ultrasónico [2]. En este segundo caso, que es el investigado en este trabajo, se produce una fina nube de gotas que se desprenden con baja velocidad. La posibilidad de generar nubes de gotas mediante ondas ultrasónicas fue inicialmente propuesta por Wood y Loomis en 1927 [2]. Para explicar este proceso, suele acudirse a dos mecanismos físicos, la formación de ondas capilares en la superficie del líquido [3,4], y la cavitación [5]. La interacción entre estos dos fenómenos, o su preponderancia en determinadas condiciones de operación es todavía un problema sin resolver. De un modo simplificado, el proceso completo para las condiciones que se han estudiado en este artículo puede resumirse como sigue. Si se sitúa una película de líquido sobre la superficie de una cerámica piezoeléctrica y se la excita con una tensión alterna a la frecuencia de resonancia, la cerámica transmite su movimiento vibratorio a las partículas del líquido. Si la fecuencia de resonancia y la amplitud de vibración son lo suficientemente importantes se crean zonas locales de depresión tan elevada que el líquido cambia de fase dando lugar a la formación de burbujas de vapor por cavitación. Dichas burbujas se dirigen hacia la superficie del fluido donde rompen su tensión superficial y generan una nube de gotitas microscópicas que se disipan en el aire.

En las últimas décadas, el desarrollo de materiales piezocerámicos ha despertado un renovado interés en el estudio de este tipo de atomizadores porque son capaces de producir aerosoles con unas características peculiares. La distribución de tamaños es muy estrecha y, en principio, el diámetro medio depende sólo de la frecuencia ultrasónica [6] y es, por tanto, fácilmente controlable. Este método resulta especialmente atractivo para generar gotas de tamaños microscópicos, difíci- 
les de conseguir por medios mecánicos, trabajando con frecuencias en el rango de MHz. Tamaños de este orden (típicamente en torno a $5 \mu \mathrm{m}$ ) son los requeridos en aplicaciones tan importantes como la nebulización e inhalación de medicamentos en solución para tratamientos de afecciones de las vías respiratorias. Aunque existen referencias que describen circuitos específicos de excitación para dispositivos piezoeléctricos [7] [8], una dificultad que se presenta en su estudio es la escasez de fuentes de señales ultrasónicas sintonizables en frecuencia y capaces de proporcionar las potencias necesarias para que se produzca la formación de gotas. En el presente trabajo se expone un sistema electrónico de alimentación para dispositivos resonadores ideado para paliar este problema y permitir la caracterización de las cerámicas fijando unas condiciones de test preestablecidas. En particular, el sistema está orientado hacia el test de resonadores que en su aplicación final serán alimentados con convertidores de potencia similares al implementado. En estas condiciones los datos obtenidos de los tests de caracterización proporcionan una valiosa información para prever el comportamiento del dispositivo atomizador en condiciones reales de trabajo. En los siguientes apartados se describirá el comportamiento eléctrico de los transductores piezoeléctricos, los circuitos que constituyen el sistema de caracterización propuesto, sus características principales, y los primeros ensayos destinados a la caracterización de un disco piezoeléctrico con la ayuda de un sistema de medida del diámetro de gota basado en un difractómetro láser.

\section{CARACTERÍSTICAS ELÉCTRICAS DEL RESONADOR}

\subsection{Circuito equivalente.}

La familia de los circoniatos-titaniatos de plomo (PZT) constituye el material base para la mayoría de transductores electromecánicos piezoeléctricos, gracias a los elevados parámetros de polarización que pueden alcanzar, así como a la posibilidad de modificarlos con la introducción de determinados aditivos. Los dispositivos destinados a la atomización de fluidos suelen presentarse bajo la forma de discos de dicho material cerámico sinterizado, con dos contactos eléctricos, uno en cada cara del disco. Desde el punto de vista eléctrico, su respuesta en frecuencia alrededor de la frecuencia de resonancia es del tipo mostrado en la Figura 1, obtenida de un analizador de espectros HP4195A para un transductor PZT de American Piezo Ceramics Inc. (Mackeyville, PA, USA). Puede observarse el abrupto cambio de fase coincidiendo con el mínimo de impedancia a la frecuencia propia de resonancia nominal en 1,668 MHz. Esta frecuencia de resonancia está asociada a un modo de vibración mecánico, responsable de la transmisión del movimiento al líquido. El comportamiento de dicha respuesta en frecuencia suele describirse mediante el circuito equivalente de la Figura 2.a, compuesta de una rama R-L-C serie, en paralelo con una capacidad Co. Este último elemento está relacionado con la capacidad estática asociada a las metalizaciones necesarias para realizar los contactos eléctricos. Sin embargo, si se aumenta la frecuencia de análisis, se observan una sucesión de cambios de fase e impedancia como el ya mostrado aunque de menor magnitud, correspondientes a frecuencias propias de resonancia de orden superior y a sus modos mecánicos de vibración. Por consiguiente, el comportamiento del dispositivo estaría descrito de un modo más exacto con un circuito equivalente como el de la Figura 2.b, con más de una rama R-L-C en paralelo con Co. La

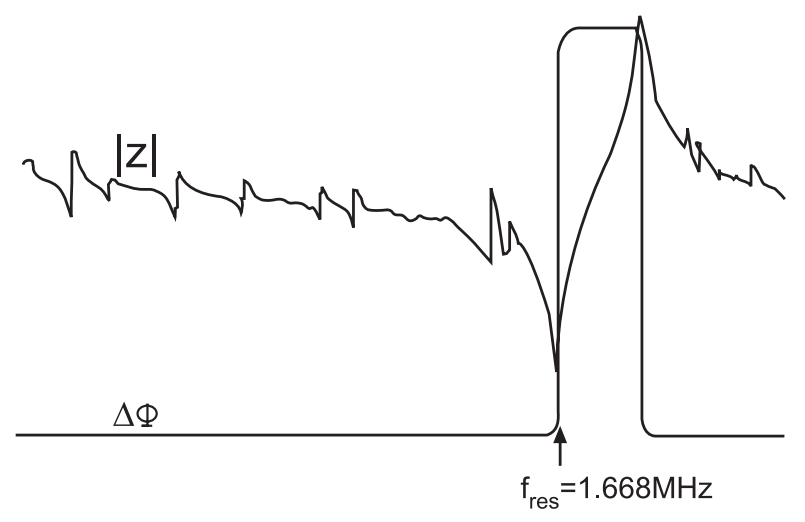

Figura 1. Gráfica de la respuesta en frecuencia de un resonador piezoeléctrico PZT alrededor de la frecuencia de resonancia de 1,668 $\mathrm{MHz}$, obtenida con el analizador de espectros.

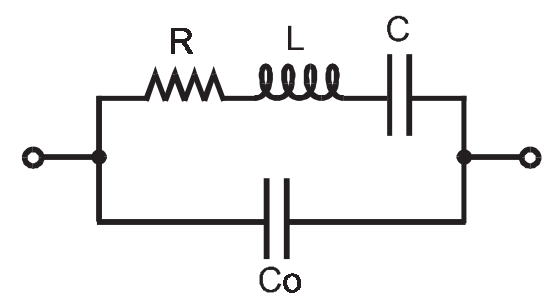

Figura 2.a. Circuito equivalente del resonador piezoeléctrico alrededor de su frecuencia propia de resonancia nominal.

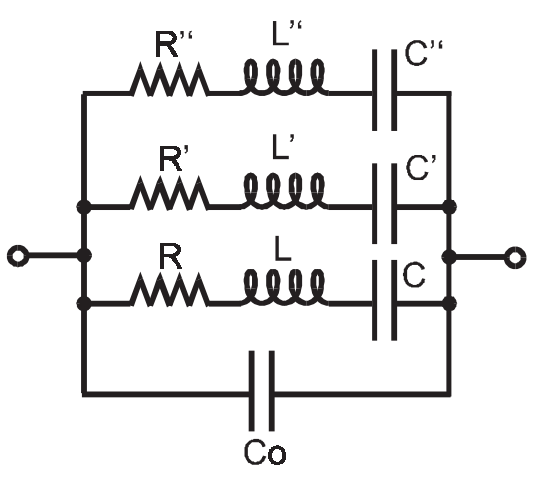

Figura 2.b. Circuito equivalente del resonador piezoeléctrico cuando se toman en cuenta tres frecuencias propias de resonancia.

TAbla I. PARÁmetros del Circuito EQUivalente DEL RESONADOR PIEZOELÉCTRICO ESTUDIADO.

\begin{tabular}{|c|c|c|c|c|}
\hline Frecuencia & $\mathrm{R}$ & $\mathrm{L}$ & $\mathrm{C}$ & $\mathrm{Co}_{0}$ \\
\hline $1,6675 \mathrm{MHz}$ & $2,2 \Omega$ & $67,26 \mu \mathrm{H}$ & $135,283 \mathrm{pF}$ & $706,499 \mathrm{pF}$ \\
\hline $5,46 \mathrm{MHz}$ & $31,15 \Omega$ & $38,644 \mu \mathrm{H}$ & $21,786 \mathrm{pF}$ & $965,796 \mathrm{pF}$ \\
\hline $15,592 \mathrm{MHz}$ & $325,59 \mathrm{~m} \Omega$ & $135,78 \mathrm{nH}$ & $767,177 \mathrm{pF}$ & $1,30483 \mathrm{nF}$ \\
\hline
\end{tabular}

Tabla I muestra los valores experimentales obtenidos de los parámetros R-L-C y Co para el transductor PZT estudiado. Puede observarse como el valor de Co varia relativamente poco cuando se extraen los valores de los demás elementos a las frecuencias propias de $5,46 \mathrm{MHz}$ y $15,592 \mathrm{MHz}$ y puede considerarse constante en primera aproximación. 


\subsection{Relaciones electromecánicas.}

Desde el punto de vista del diseño del circuito de excitación, es suficiente con conocer el comportamiento eléctrico de la cerámica piezoeléctrica proporcionado por su circuito equivalente. Sin embargo, es interesante también conocer la relación entre la potencia y la frecuencia suministradas al transductor y la atomización del fluido en contacto con él. Por una parte, el diámetro promedio $\mathrm{D}(\mathrm{en} \mathrm{cm})$ de las gotas atomizadas viene dado por la expresión de Lang [6]:

$$
\mathrm{D}=0.34\left(\frac{8 \pi \mathrm{T}}{\rho \mathrm{f}^{2}}\right)^{\frac{1}{3}}
$$

Donde $\mathrm{T}$ representa la tensión superficial del fluido, $\rho$ su densidad y f la frecuencia de excitación. Así pues, y como ya se ha mencionado, para un fluido determinado el tamaño de gota que se genera por atomización ultrasónica tan sólo depende de la frecuencia de resonancia del transductor. Por otra parte, la potencia P necesaria para atomizar un flujo volúmico $\mathrm{D}_{\mathrm{v}}$ de líquido viene dada por la expresión aproximada [9]:

$$
\mathrm{P}=\frac{3 \mathrm{~T} \mathrm{D}_{\mathrm{v}}}{0.5 \mathrm{D}}
$$

Experimentalmente se ha determinado que esta potencia representa aproximadamente el $8 \%$ de la potencia total ultrasónica y el resto se pierde en reflexiones múltiples en el fluido, fenómenos no lineales, etc. Una parte de la energía eléctrica cedida al resonador se disipa también en forma de calor en la resistencia que aparece en el circuito equivalente. De todos modos, el campo de la atomización ultrasónica es complejo, continúa abierto y las expresiones anteriores se ven a menudo replanteadas y corregidas.

\section{DISEÑO DEL SISTEMA DE EXCITACIÓN}

El objetivo de un circuito de excitación para un transductor piezoeléctrico, consiste en la aplicación de una tensión alterna entre sus bornes, transmitiéndole al mismo tiempo la potencia necesaria para la formación de la nube de gotas. Las partes esenciales de que se compondrá un sistema de este tipo son la etapa de potencia, directamente conectada al transductor y responsable de transmitirle la potencia necesaria desde una fuente de energía primaria, la etapa de control encargada de gestionar el funcionamiento de la etapa de potencia así como de realizar la interfase con el usuario y finalmente, las fuentes de alimentación auxiliares. Para evitar el autocalentamiento del piezoeléctrico el sistema debe poder excitarse en modo ráfaga, es decir, enviando un numero $\mathrm{N}$ de pulsos de tensión a la frecuencia deseada tras los cuales se deja a la cerámica en reposo hasta la llegada de la nueva ráfaga. El número de pulsos, su frecuencia, su ciclo de trabajo, su amplitud y la frecuencia de repetición de la ráfaga deben ser controlables. Los transductores que se pretenden estudiar están destinados a aplicaciones de atomización donde se obtengan tamaños de gota del orden de algunas micras, lo que se traduce en frecuencias de excitación de entre varios centenares de $\mathrm{kHz}$ y $2 \mathrm{MHz}$.

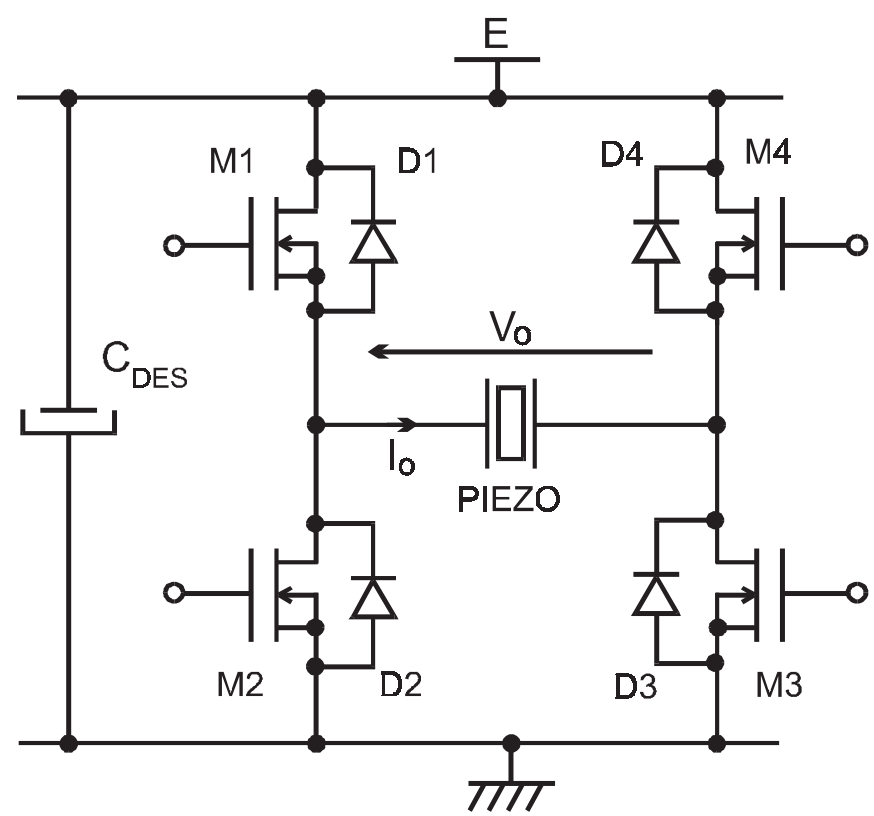

Figura 3. Esquema básico de un puente completo con transistores MOSFET.

\subsection{La etapa de potencia. El convertidor DC/AC.}

Si se considera la utilización del transductor alrededor de la frecuencia de resonancia, puede describirse su comportamiento eléctrico por medio del circuito equivalente de la Figura 2.a. Este tipo de circuito R-L-C, constituye la carga típica de la familia de convertidores de potencia llamada resonante [10] y resulta pues natural interesarse por ellos. Además, un convertidor de este tipo sería perfectamente indicado para excitar un transductor piezoeléctrico en una aplicación práctica. Existe un gran número de topologías resonantes, aunque la más versátil y que ofrece mayores posibilidades es la denominada "puente completo" o "puente en $\mathrm{H}^{\prime}$. La Figura 3 muestra el esquema básico de este tipo de convertidor implementado con transistores MOSFET y diodos en antiparalelo y que tiene como fuente primaria de energía una fuente de tensión continua E. Como en la práctica totalidad de convertidores electrónicos de potencia, los transistores trabajan en modo interruptor para evitar un excesivo autocalentamiento y se hallan siempre en dos estados posibles: en conducción o estado "ON" circula un elevado nivel de corriente y la caída de tensión en el transistor es baja, y en bloqueo o estado "OFF" la corriente es prácticamente cero y el transistor soporta una tensión relativamente elevada entre sus bornes. El control de "bloqueo" y de "disparo" de los transistores se realiza en "diagonal", es decir, las mismas señales de control se envían por parejas a M1 - M3 y M2 - M4. Además, debe evitarse la conducción simultánea de los dos transistores de una misma rama (M1 y M2 o M3 y M4), pues ello cortocircuitaría la fuente de tensión E. Ello se consigue por una parte enviando señales de control complementarias a M1 - M3 y M2 - M4 y por otra, retrasando un cierto tiempo, denominado "tiempo muerto", las señales de disparo. Con las anteriores normas en mente, el convertidor de la Figura 3 con una carga R-L-C conectada a su salida sólo puede hallarse en alguno de 
los estados de la Tabla II. Si la frecuencia de conmutación de los transistores, es ligeramente superior a la de resonancia del piezoeléctrico, las formas de onda idealizadas que se obtienen son las de la Figura 4. De ellas pueden realizarse tres observaciones de gran relevancia. En primer lugar, la tensión de salida es una forma de onda alterna, prácticamente cuadrada y con un valor pico a pico de 2E. Este punto es precisamente uno de los que hacen atractivo al puente completo, pues existen otras configuraciones que utilizan sólo uno o dos transistores pero que permiten un peor aprovechamiento de la fuente de tensión continua pues el valor pico a pico de la salida es sólo E. El segundo punto interesante que se desprende de la Figura 4 es que la corriente que atraviesa la cerámica piezoeléctrica es también alterna y sinusoidal. Además se observa que la presencia de los diodos en antiparalelo denominados de free-wheeling es indispensable para no interrumpir la circulación de la corriente en determinados instantes, en particular durante los tiempos muertos. El último punto destacable es que las conmutaciones de disparo de los transistores se realizan cuando su caída de tensión es prácticamente nula. Esta condición de operación del convertidor se denomina ZVS (Zero Voltage Switching) y presenta la ventaja de que no se producen pérdidas de potencia asociadas al proceso de puesta en conducción del MOSFET, limitándose su autocalentamiento y aumentando la frecuencia máxima de trabajo del convertidor. Hasta aquí se han discutido los aspectos más teóricos o de principio referentes al convertidor DC/AC en puente completo y en condiciones ZVS alimentando un resonador piezoeléctrico, pero su implementación requiere una serie de consideraciones prácticas de gran importancia.

Los transistores seleccionados para implementar la etapa de potencia son del tipo VDMOS (Vertical Double-diffused MOS), pues este tipo de componente es capaz de conmutar mucho más rápidamente que sus homólogos en tecnología bipolar. Además, la estructura VDMOS presenta de manera natural un diodo interno en antiparalelo que en muchas ocasiones puede ser usado como diodo de free-wheel [11]. Así pues, la selección del transistor es clave para determinar las características del sistema final. El dispositivo escogido ha sido el IRFU110 de International Rectifier, transistor de 100V de tensión de ruptura, $0.54 \Omega$ de resistencia en estado conductor y unos tiempos de conmutación del orden de los 20ns para el MOSFET y del orden de los 100ns para el diodo interno, que también puede ser utilizado. El encapsulado de este dispositivo es del tipo TO-251AA con una capacidad de disipación de potencia limitada a unos $25 \mathrm{~W}$. La idea de todo el sistema consiste en caracterizar la formación de gotas con el transductor piezoeléctrico, fenómeno que se ve alterado por la temperatura que alcance este último, por lo que para limitar su propio autocalentamiento en funcionamiento normal, se le enviarán ráfagas de entre unos pocos pulsos de tensión alterna y algunas decenas de miles. Esta manera de proceder limita también el autocalentamiento de los propios dispositivos de potencia (fenómeno que degradaría sus características), haciéndose innecesaria su refrigeración con radiadores y/o ventiladores.

Para controlar los procesos de disparo y de corte de los transistores, es necesario aplicar rápidos cambios de tensión entre 0 y $15 \mathrm{~V}$ a la puerta de los mismos, cargando y descargando su capacidad parásita con picos de corriente que pueden alcanzar hasta 0,5A y algunas decenas de ns de duración. Para realizar esta función se requiere un circuito integrado de control de puerta o driver, con una excepcional respuesta en frecuencia como la del MAX4420 que finalmente se ha usado.
TABLA II. ESTADOS POSIBLES DE UN PUENTE COMPLETO CON UNA CARGA RL-C A LA SALIDA.

\begin{tabular}{|c|c|c|c|c|c|}
\hline M1 - M3 & M2 - M4 & D1 - D3 & D2 - D4 & Iout & Vout \\
\hline ON & OFF & OFF & OFF & + & E \\
\hline OFF & OFF & OFF & ON & + & -E \\
\hline OFF & ON & OFF & ON & + & $-\mathrm{E}$ \\
\hline ON & OFF & ON & OFF & - & E \\
\hline OFF & OFF & ON & OFF & - & E \\
\hline OFF & ON & OFF & OFF & - & -E \\
\hline
\end{tabular}

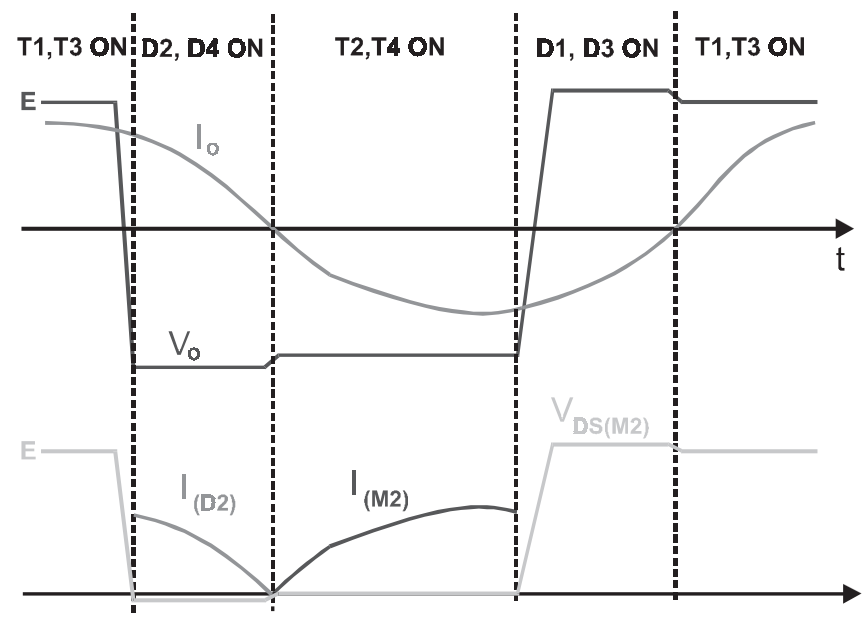

Figura 4. Formas de onda idealizadas del puente completo excitando a una carga R-L-C a una frecuencia ligeramente superior a la de resonancia.

La etapa de potencia debe estar aislada galvánicamente de la de control para proteger esta última en caso de mal funcionamiento y para permitir el control de las puertas de los MOSFET con referencias de tensión flotantes. La solución más práctica consiste en utilizar optoacopladores, pero estos dispositivos suelen ser lentos e introducen tiempos de propagación muy elevados. Finalmente, se ensayó el optoacoplador HCPL2212 de Agilent con éxito, aunque al límite de sus posibilidades. En efecto, su salida presenta unos frentes de subida de unos 100ns, que posteriormente son reconstituidos por el driver MAX4420, además de introducir un retraso global de $125 \mathrm{~ns}$ que no supone ningún problema pues se da por igual en los cuatro canales de control, uno por cada transistor. Otras característica importantes del HCPL2212 son su capacidad de operar con tensiones de alimentación de $15 \mathrm{~V}$ y su elevado CMMR (Common Mode Rejection Ratio), parámetro que garantiza un buen aislamiento hacia la etapa de control del ruido generado por los elevados dV/dt en la potencia. Finalmente, la etapa de potencia necesita de una fuente primaria de energía en forma de fuente de tensión continua, que para proporcionar mayor flexibilidad y tratándose de un sistema de laboratorio, puede ser cualquier fuente externa que no sobrepase los 100V. Justo a la entrada del puente en $\mathrm{H}$, la tensión continua de potencia debe ser desacoplada con una capacidad que evite un elevado rizado de la misma cuando sea solicitada con 
picos de corriente elevados. Cada canal constituido por un optoacoplador, un driver y un MOSFET está dotado con una fuente de alimentación aislada de $15 \mathrm{~V}$, tensión que aplicada a la puerta del MOSFET proporciona una resistencia en conducción suficientemente baja, así como una buena inmunidad frente a picos parásitos de tensión. Toda la etapa de potencia ha sido realizada en una misma placa de circuito impreso y dada la elevada frecuencia de trabajo de los transistores así como de los niveles de corriente que pueden alcanzarse (algunos amperios), el layout ha sido muy cuidado, principalmente con el objetivo de minimizar inductancias parásitas responsables de picos no deseados de tensión.

\subsection{La etapa de control.}

La etapa de control consta esencialmente de la placa con los circuitos de control y de los mandos de panel frontal que fijan las condiciones de test. Dicha placa de control está alimentada a $5 \mathrm{~V}$ pues para implementar las funciones necesarias se han utilizado puertas lógicas CMOS de las familias 74HCXX y 74ACXX capaces de trabajar holgadamente a las frecuencias requeridas. La generación de los diversos impulsos que darán origen a las señales de control de la etapa de potencia se han confiado a la versión CMOS del conocido timer 555 que alcanza los $2 \mathrm{MHz}$ sin dificultad. Como puede verse, la filosofía de diseño ha sido utilizar circuitos integrados discretos y de bajo coste en lugar de microcontroladores, DSP o circuitos lógicos programables que requieren costosos sistemas de desarrollo y programación. Ello ha sido motivado por la relativa sencillez de las señales que debe generar el sistema y por la búsqueda de un sistema robusto y fácil de reparar. El circuito de control, funciona de la manera siguiente. Dos circuitos timer 555 en configuración astable generan la frecuencia de salida y la de repetición de las ráfagas en función de los potenciómetros correspondientes en el panel frontal. Un tercer potenciómetro fija el ciclo de trabajo de la tensión alterna de salida gracias a un tercer integrado 555 en configuración monoestable. Unos preselectores de panel frontal establecen el número de pulsos que constituirán la ráfaga. Este número en código BCD es comparado en un bloque de comparadores con el número actual de pulsos que se dirigen a la salida de la placa de control, y cuando se alcanza el número preestablecido, se activa un subcircuito que inhabilita la posterior generación de pulsos hasta la ráfaga siguiente. Finalmente, a las cuatro señales de control correspondientes a los cuatro transistores de la etapa de potencia se les retrasan los frentes de subida para generar los tiempos muertos que evitan la conducción simultánea transitoria de dos transistores de una misma rama del puente. Para los transistores IRFU110 utilizados, un tiempo muerto de 120ns ha sido suficiente. La Figura 5, muestra una fotografía del panel frontal del sistema, donde a parte de los potenciómetros y preselectores ya mencionados, se pueden observar los conectores para la fuente de alimentación continua, los de salida de la etapa de potencia con el transductor PZT conectado, unos conectores BNC que proporcionan las señales de control del puente en $\mathrm{H}$ y la posibilidad de medir la frecuencia de excitación, y unos interruptores de disparo de las ráfagas y de habilitación del sistema en modo de ráfaga única o repetitiva. En resumen, las prestaciones y condiciones de test que permite establecer el sistema diseñado son las siguientes:

- Frecuencia de oscilación del tren de impulsos que constituye una ráfaga, entre $500 \mathrm{kHz}$ y $2 \mathrm{MHz}$.

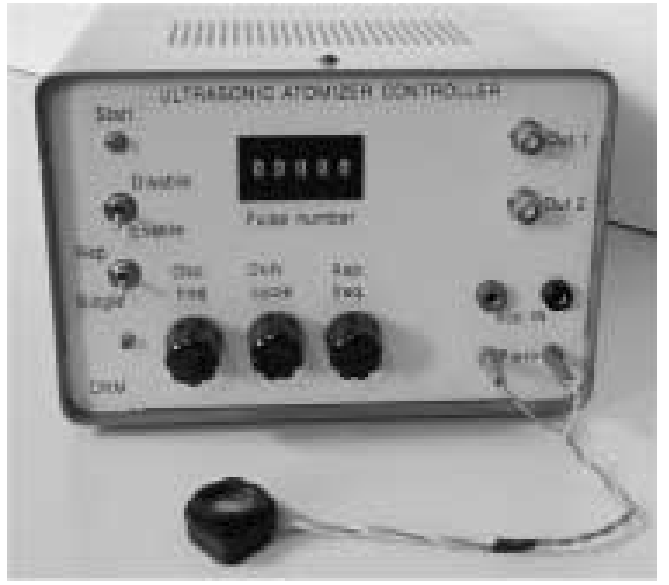

Figura 5. Fotografía del panel frontal del sistema de excitación de resonadores piezoeléctricos con el transductor PZT de $1,668 \mathrm{MHz}$ conectado a la salida.

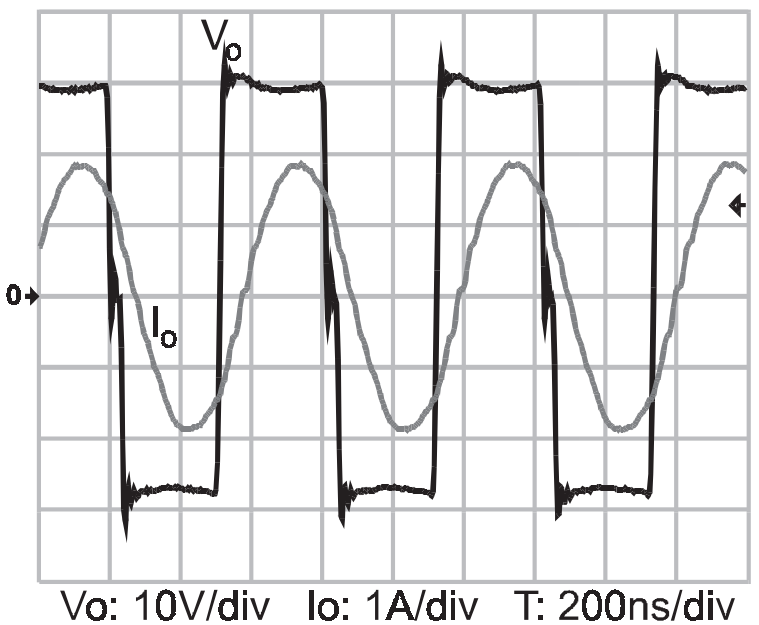

Figura 6. Formas de onda con una carga R-L-C pura, reproduciendo el circuito equivalente del resonador piezoeléctrico de 1,668MHz.

- Frecuencia de repetición de las ráfagas entre $1 \mathrm{~Hz}$ y $1 \mathrm{kHz}$.

- Número de pulsos que componen la ráfaga entre 1 y 99.999.

- Elección entre un modo de ráfaga única disparada manualmente o una sucesión repetitiva de ráfagas.

\section{RESULTADOS Y DISCUSIÓN}

\subsection{Resultados eléctricos}

Tras los lógicos ensayos de validación funcional del equipo de test, los primeros ensayos en carga se realizaron con un circuito R-L-C construido con componentes discretos que reproducían el circuito equivalente de la Figura 2.a para el resonador descrito en el apartado 2.1, con una frecuencia de resonancia cercana a 1,7MHz. En la Figura 6 pueden observarse las formas de onda de la tensión en bornes de la carga y de la corriente que la atraviesa cuando se aplica una tensión de excitación con una frecuencia ligeramente superior a la de reso- 
nancia. La respuesta del circuito es casi ideal y se ha comprobado que al añadir la capacidad estática Co, se observa una ligera disminución de los frentes de subida de la tensión y del valor máximo de la corriente, sin mayores consecuencias. A nivel práctico, los efectos de esta capacidad se pueden minimizar conectándole una inductancia en paralelo para que ambos componentes constituyan un circuito "tapón" a la frecuencia de resonancia nominal de la cerámica. Al conectar el resonador piezoeléctrico a la salida de la etapa de potencia con una tensión continua $\mathrm{E}=45 \mathrm{~V}$, las formas de onda de la tensión y de la corriente que se obtuvieron, son las de la Figura 7. Pueden realizarse dos observaciones evidentes. En primer lugar, la corriente ya no es prácticamente sinusoidal y en segundo lugar, las formas de onda de la tensión no son prácticamente cuadradas y tienen superpuestas otras oscilaciones de mayor frecuencia. Tras un análisis de los resultados anteriores, se concluyó que estos efectos son debidos a la excitación de las frecuencias propias de resonancia de orden superior al nominal, debido a las elevadas velocidades de variación de la tensión de salida. Este punto fue corroborado al simular con el programa PSPICE el puente completo alimentando al circuito equivalente de la Figura 2.b. La corriente total que atraviesa la cerámica es la suma de las corrientes de cada una de las ramas R-L-C (la nominal de $1,668 \mathrm{MHz}$, la de $5,46 \mathrm{MHz}$ y la de $15,592 \mathrm{MHz}$ ), más la de Co, siendo el resultado muy similar a la forma de onda experimental de la Figura. 7.

\subsection{Medidas del tamaño de gota}

Tras la caracterización eléctrica del sistema, se observó que la influencia de los modos propios de orden superior no afecta a la capacidad de atomización del equipo, aunque probablemente si debe disminuir su eficiencia. Así pues, se procedió a un primer estudio del proceso de atomización con la cerámica PZT y el sistema de excitación diseñado. La frecuencia de salida de la etapa de potencia debe ser cuidadosamente ajustada hasta alcanzar la de resonancia nominal del transductor, momento en que puede empezar a observarse la atomización. Sin embargo, son necesarios un mínimo de 10.000 pulsos para iniciar la formación de gotas con tensiones continuas aplicadas entre 30 y $60 \mathrm{~V}$, que corresponden a tensiones pico a pico de entre 60 y $120 \mathrm{~V}$ respectivamente. Por debajo de este valor tan sólo se observa un movimiento en la superficie del líquido. Otro parámetro que conviene ajustar a valores cercanos al $50 \%$ es el ciclo de trabajo de la tensión alterna de salida, pues de otro modo se pierde rápidamente eficacia en la atomización. Teniendo en cuenta estos puntos, el equipo ha sido utilizado en ensayos de medida de tamaño de gota, integrándolo en un sistema de difractometría láser Malvern MasterSizer como se aprecia en la Figura 8. Los resultados de estos ensayos se pueden observar sintetizados en la gráfica de la Figura 9. Puede observarse como la distribución relativa de diámetros de gota varía con tensiones continuas aplicadas de 50,55 y $60 \mathrm{~V}$, pero el máximo se mantiene inamovible alrededor de las $9 \mu \mathrm{m}$, dependiendo tan sólo de la frecuencia de excitación como predice la teoría básica de la atomización ultrasónica.

\section{CONCLUSIONES}

El estudio de transductores piezoeléctricos destinados a la atomización de fluidos para generar nubes de gotas microscópicas, presenta el problema de la disponibilidad de fuentes de

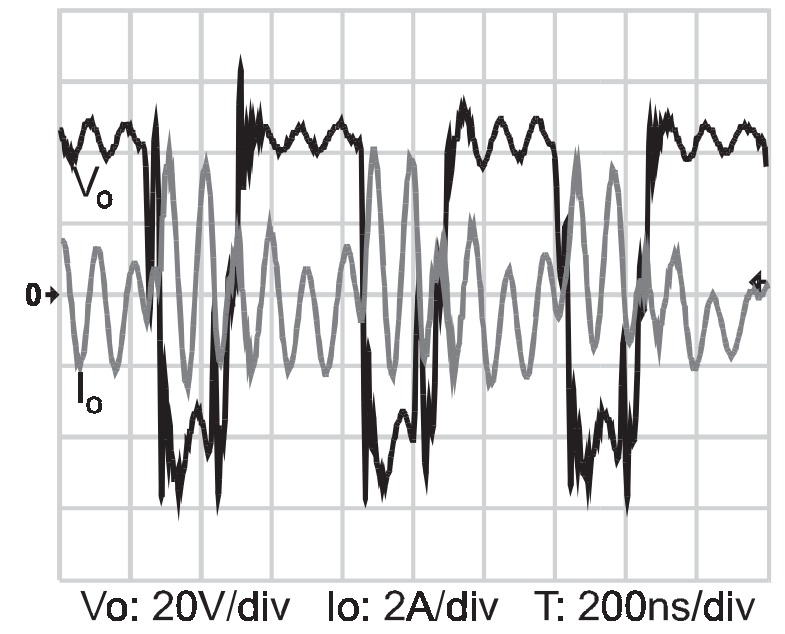

Figura 7. Formas de onda de tensión y corriente del resonador piezoeléctrico de $1,668 \mathrm{MHz}$ conectado a la salida del equipo de test.

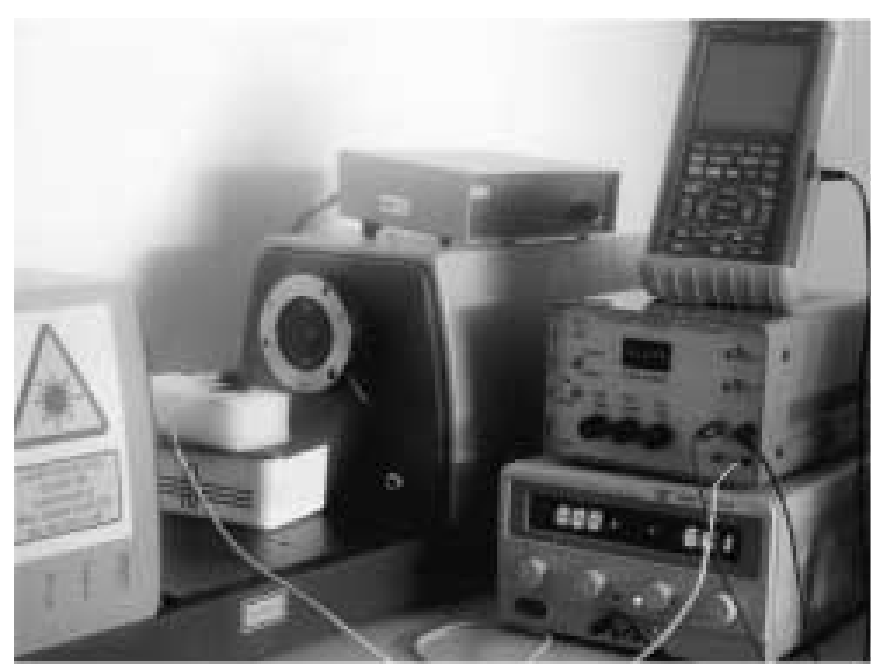

Figura 8. Fotografía del equipo de excitación de resonadores piezoeléctricos, integrado en un sistema de medida de tamaño de gota por difractometría láser.

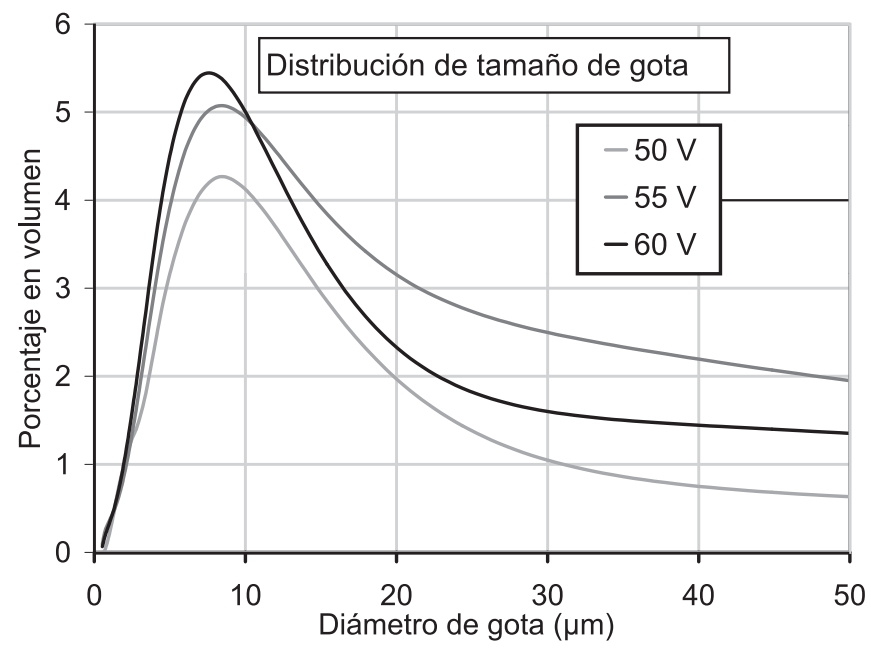

Figura 9. Curvas de distribución del diámetro de gota para tres tensiones continuas de entrada. En los tres casos el máximo se sitúa sobre las $9 \mu \mathrm{m}$. 
excitación de tensión alterna con frecuencia de salida variable y capaz de proporcionar la energía necesaria al transductor. En este trabajo se ha realizado un sistema electrónico que cumple dicha función. El núcleo del sistema lo constituye un convertidor DC/AC con topología de puente completo conmutando en modo ZVS con cargas tipo R-L-C. El circuito equivalente de un resonador piezoeléctrico es precisamente de este tipo por lo que el convertidor utilizado parece óptimo para esta aplicación. Se ha comprobado sin embargo que las rápidas variaciones de tensión que se producen a la salida del convertidor excitan las frecuencias propias de orden superior de la cerámica y la corriente que en un principio debía ser sinusoidal es en realidad la superposición de por lo menos tres componentes armónicas. El circuito equivalente que debería pues considerarse, añade dos ramas R-L-C adicionales en paralelo con la principal. Todo ello no impide en absoluto que el transductor atomice correctamente, lo que se ha puesto de manifiesto durante los ensayos de medida de tamaño de gota con un difractómetro láser correspondientes a un disco de cerámica PZT con una frecuencia de resonancia de $1,668 \mathrm{MHz}$. Se ha podido estudiar la influencia de parámetros de test como el número mínimo de pulsos necesarios para iniciar la atomización (10.000), la frecuencia de excitación (la de resonancia es, evidentemente, la óptima), el ciclo de trabajo (50\% es el valor óptimo) y la tensión pico a pico aplicada al resonador (60V como mínimo). Se observa que la distribución del tamaño de gota varia con la tensión aplicada aunque siempre existe un máximo alrededor de las $9 \mu \mathrm{m}$ de diámetro. El sistema está pensado para funcionar en modo ráfaga, excitando al piezoeléctrico con un número $\mathrm{N}$ de pulsos a la frecuencia de resonancia, evitando así su autocalentamiento y consiguientemen- te los cambios de características en función de la temperatura e incluso la despolarización de la cerámica. La configuración y el uso del sistema resultan muy sencillos, es prácticamente autónomo, requiriendo tan sólo una fuente de tensión continua externa, muy robusto, de bajo coste y fácil mantenimiento pues se basa en circuitos y componentes estándar.

\section{BIBLIOGRAFÍA}

1. L. Bendig, "New developments of ultrasonic atomizers", Proceedings of the 4th Int. Conf. Liquid Atomization and Sprays Systems, 133-138, (1988).

2. W. R. Wood, A.L. Loomis. "The physical and biological effects of high frequency sound-waves of great intensity", Phil. Mag. vol. 4 (22), 417-437, (1927).

3. M. Faraday, "On the forms and states assumed by fluids in contact with vibrating elastic surfaces", Phil. Trans. R. Soc. Lond. 52, 319-340, (1831).

4. Lord Rayleigh "On the crispation of fluid resting upon a vibrating suppor", Phil. Mag. 15, (5), 50-58, (1883).

5. K. Söllner, "The Mecanism of the Formation of Fogs by Ultrasonic Waves", Trans. Faraday Soc., 32, 1532-1536, (1936).

6. R.J. Lang. “Ultrasonic Atomization of Liquids", J. Acoust. Soc. Am., vol. 34 (1), 6-8, (1962)

7. E. Minguella, C. Segura, A. Albareda, R. Perez. "Inversor a 700kHz para un vaporizador piezoeléctrico", Congreso SAAEI'98, Pamplona, 1998. p. 213215.

8. T. Zaitsu, T. Shigehisa, M. Shoyama, T. Ninomiya. "Piezoelectric Transformer Converter with PWM Control" IEEE APEC Conference, 1996, p. 279-283.

9. A. Giovannini, D. Guyomar, M. Gschwind, G. Fonzes. "Evaluation and design of new piezoelectrical droplets generator", IEEE Ultrasonics Symposium, 1994, p.611-614.

10. N. Mohan, T. M. Undeland, W. P. Robbins. "Power Electronics: Converters, Applications and Design" John Whiley and Sons, New York, 1989.

11. D. A. Grant, J. Gowar. "Power Mosfets. Theory and Applications" John Whiley and Sons, New York, 1989.

Recibido: 31.05 .01

Aceptado: 10.12 .01 